\title{
A GENERALIZATION OF A THEOREM OF LEROY AND LINDELÖF
}

\author{
V. F. COWLING
}

1. Introduction. Consider a Taylor series $f(z)=\sum_{n=0}^{\infty} a_{n} z^{n}$ with radius of convergence unity. Let the coefficients $a_{n}$ be the values taken on by a regular function $a(z)$ for $z=0,1, \cdots$.

The object of this paper is to study the Taylor series under the assumption that $a(z)$ is regular in certain domains. ${ }^{1}$ The results obtained are of the nature of domains in which the function defined by $\sum_{n=0}^{\infty} a_{n} z^{n}$ is regular and of domains which contain the singularities of the function defined by the series. In terms of $a(z)$ fairly general sufficient conditions are given such that the circle of convergence is not a cut for the function.

The results may be regarded as a generalization of a theorem due to LeRoy and Lindelöf. ${ }^{2}$

TheOREM (LeRoy AND LiNDELöF). Suppose (a) $a(x+i y)$ is regular in the semiplane $x \geqq \alpha$, (b) there is a $\theta<\pi$ such that for every arbitrary small positive $\epsilon$ and for sufficiently large $\rho$

$$
|a(\alpha+\rho \exp (i \psi))|<\exp [\rho(\theta+\epsilon)], \quad-\pi / 2 \leqq \psi \leqq \pi / 2 .
$$

Then

$$
f(z)=\sum_{n=0}^{\infty} a(n) z^{n}, \quad z=r \exp (i \phi)
$$

is regular in the angle

$$
\theta<\phi<2 \pi-\theta \text {. }
$$

The generalization of this theorem that we prove consists, under suitable restrictions, in replacing the semiplane $x \geqq \alpha$ by an angular opening including the axis of positive reals in its interior.

The singularities of the function $f(z)$ studied in this paper are those of a "principal branch" obtained by immediate continuation of the series.

Consider an angular opening with vertex on the positive real axis which includes the axis of reals in its interior. Suppose $a(z)$ has no singularities in this angular opening with the possible exception of

Presented to the Society, December 29, 1946; received by the editors May 29, 1946.

1 By the term domain we mean an open connected set.

${ }^{2}$ See Dienes [1]. Numbers in brackers refer to the bibliogiaphy at the end of the paper. 
the point at infinity. Let the sides of the angular opening make angles $\psi_{1}$ and $\psi_{2}$ with the axis of reals.

Our problem is to characterize the behavior of the function $f(z)$ in terms of the magnitudes of the angles $\psi_{1}$ and $\psi_{2}$ and the type of singularity $a(z)$ has at infinity.

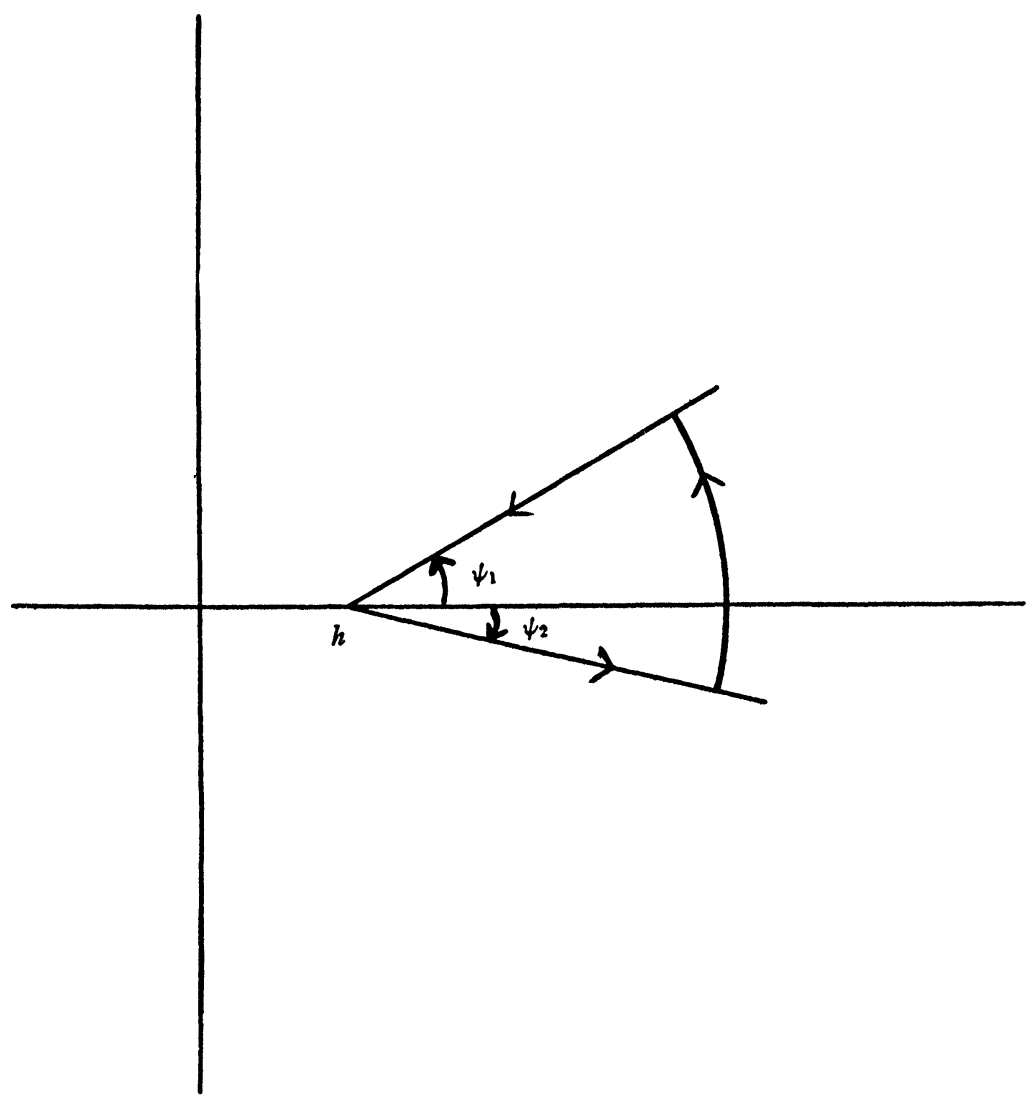

FIG. 1

We consider first the case where $a(z)$ may have a pole of order $K$ at infinity and then the case in which infinity may be an essential singularity for $a(z)$.

2. $a(z)$ may have a pole at infinity. Suppose now that $a(z)$ is regular interior to and on the sides of the angular opening shown in Figure 1, except for at most a pole of order $K$ at infinity. In Figure 1 let $l-1<h<l$ where $l$ is a positive integer.

By the calculus of residues if $F(\omega)$ and $G(\omega)$ are uniform functions 
in a domain and if $G(\omega)$ has only simple zeros $\alpha_{i}$ in this domain, then (integration being understood in the positive sense)

$$
\int_{C} \frac{F(\omega)}{G(\omega)} d \omega=\frac{F\left(\alpha_{i}\right)}{G^{\prime}\left(\alpha_{i}\right)}
$$

where $C$ is a path enclosing $\alpha_{i}$ but no other zero of $G(\omega)$ and $G^{\prime}\left(\alpha_{i}\right)$ is the derivative of $G(\omega)$ evaluated at $\omega=\alpha_{i}$.

Let $F(\omega)=a(\omega) z^{\omega}$ and $G(\omega)=\exp (2 \pi i \omega)-1$.

For a given value of $z=r \exp (i \theta)$ we shall understand by $z^{\omega}$

$$
z^{\omega}=\exp [\omega(\log r+i \theta)], \quad 0 \leqq \theta<2 \pi .
$$

Here $\exp z$ is the principal value of $e^{z}$. This convention will be adhered to throughout the paper.

Then by (1)

$$
\frac{1}{2 \pi i} \int_{C} \frac{a(\omega) z^{\omega}}{\exp (2 \pi i \omega)-1} d \omega=a(n) z^{n}
$$

where $C$ is a path enclosing $n$ and no other real integer. $a(\omega)$ is a function regular in the angular opening. This choice of $F(\omega)$ and $G(\omega)$ led to the well known theorem of LeRoy and Lindelöf [1] and is of course a well known method for the summation of certain series. The analysis of this paper follows lines similar to those in the analysis of Lindelöf.

Consider

$$
\int_{C_{h}, R} \frac{a(\omega) z^{\omega}}{\exp (2 \pi i \omega)-1} d \omega
$$

where $C_{h, R}$ is the path formed by the two sides of the angular opening, with vertex $h>0$, and the arc of a circle of radius $R$, where $R$ is a positive integer. This is indicated in Figure 1.

By application of (2) it follows that

$$
\frac{1}{2 \pi i} \int_{C_{h}, R} \frac{a(\omega) z^{\omega}}{\exp (2 \pi i \omega)-1} d \omega=\sum_{n=l}^{R+l-1} a(n) z^{n} .
$$

Denote the sides of the angular opening corresponding to $\psi_{1}$ and $\psi_{2}$ by $I_{1}$ and $I_{2}$ and the arc of a circle by $C$. Then

$$
\begin{aligned}
& \int_{C_{h}, R} \frac{a(\omega) z^{\omega}}{\exp (2 \pi i \omega)-1} d \omega=\int_{I_{2}} \frac{a(\omega) z^{\omega}}{\exp (2 \pi i \omega)-1} d \omega \\
& \quad+\int_{C} \frac{a(\omega) z^{\omega}}{\exp (2 \pi i \omega)-1} d \omega+\int_{I_{1}} \frac{a(\omega) z^{\omega}}{\exp (2 \pi i \omega)-1} d \omega .
\end{aligned}
$$


It will now be shown that, if we place certain restrictions on $\psi_{1}, \psi_{2}$ and $z$,

$$
\int_{c} \frac{a(\omega) z^{\omega}}{\exp (2 \pi i \omega)-1} d \omega
$$

converges uniformly to zero as $R$ becomes infinite.

Let $z=r \exp (i \pi)$ and $\omega=h+R \exp (i \psi)$ with $-\pi<v \leqq \psi \leqq u<\pi$. By hypothesis there exists an $R_{0}$ such that in the angular opening with vertex $h>0$

$$
|a(h+R \exp (i \psi))|<A R^{K}, \quad R>R_{0},
$$

where $A$ is a constant and $K$ is a positive integer. On the arc $C$

$$
\left|\frac{1}{\exp (2 \pi i \omega)-1}\right|<B
$$

where $B$ is a constant. This follows from the fact that $\omega$ is bounded away from an integer by our choice of $h, l$ and $R$.

Clearly

$$
\left|\frac{1}{\exp (2 \pi i \omega)-1}\right|=\left|\frac{1}{1-\exp (-2 \pi i \omega)}\right||\exp (-2 \pi i \omega)| .
$$

Hence on $C$,

$$
\left|\frac{1}{\exp (2 \pi i \omega)-1}\right|<D \exp (2 \pi R \sin \psi),
$$

where $D$ is a constant. This inequality proves useful for $-\pi<v \leqq \psi<0$. Now

$$
\left|\int_{C} \frac{a(\omega) z^{\omega}}{\exp (2 \pi i \omega)-1} d \omega\right| \leqq \int_{C} \frac{|a(\omega)|\left|z^{\omega}\right|}{|\exp (2 \pi i \omega)-1|}|d \omega|
$$

and

$$
\begin{aligned}
\left|z^{\omega}\right| & =|\exp [(h+R \cos \psi+i R \sin \psi)(\log r+i \pi)]| \\
& =r^{h} \exp \left[-R\left(\log r^{-1} \cos \psi+\pi \sin \psi\right)\right] .
\end{aligned}
$$

If $r<1$, then

$$
b(r, \psi)=\log r^{-1} \cos \psi+\pi \sin \psi>0,
$$$$
0 \leqq \psi \leqq \pi / 2 \text {. }
$$

If $r>\exp (\pi \tan \psi)$, then

$$
b_{1}(r, \psi)=\log r^{-1} \cos \psi+\pi \sin \psi>0, \quad \pi / 2 \leqq \psi \leqq u<\pi .
$$

Clearly $r>\exp (\pi \tan u)$ is sufficient for (10) to hold. We note here 
and also in (11) below, that the case $|\psi|=\pi / 2$ is included. If $r<1$ then

$$
b_{2}(r, \psi)=\log r^{-1} \cos \psi-\pi \sin \psi>0, \quad-\pi / 2 \leqq \psi \leqq 0 .
$$

If $r>\exp (-\pi \tan \psi)$, then

$$
b_{3}(r, \psi)=\log r^{-1} \cos \psi-\pi \sin \psi>0, \quad-\pi<v \leqq \psi \leqq-\pi / 2 .
$$

Clearly $r>\exp (-\pi \tan v)$ is sufficient for (12) to hold.

We have seen that if $-\pi / 2 \leqq \psi \leqq \pi / 2, r<1$ implies $b(r, \psi)>0$ and $b_{2}(r, \psi)>0$. Denote, for $\alpha>0$ but otherwise arbitrarily small, by $r_{1}$ the larger of $\exp (\pi \tan u)+\alpha$ and $\exp (-\pi \tan v)+\alpha$. Set $r_{2}=1-\alpha^{\prime}$, $\alpha^{\prime}>0$ but otherwise arbitrarily small. Then $r_{1} \leqq r \leqq r_{2}$ implies $\pi / 2<u$ $<\pi$ and $-\pi<v<-\pi / 2$. We choose $\alpha$ and $\alpha^{\prime}$ sufficiently small, so that $r_{1} \leqq r \leqq r_{2}$ is an interval consisting of more than one point. Then, for a given $\alpha$ and $\alpha^{\prime}$ and $r_{1} \leqq r \leqq r_{2},(9),(10)$, (11), and (12) take on their minimum values on their respective intervals of definition. Let these be $b^{\prime}>0, b_{1}^{\prime}>0, b_{2}^{\prime}>0$ and $b_{3}^{\prime}>0$. Denote by $\beta>0$ the smallest of these four values. Then by application of (5), (6) and (7) for $R>R_{0}$, (8) becomes

$$
\begin{aligned}
\left|\int_{c} \frac{a(\omega) z^{\omega}}{\exp (2 \pi i \omega)-1} d \omega\right| & \leqq T_{0}(A, B, D) R \int_{v}^{u} \exp (-\beta R) d \psi \\
& =T_{0}(A, B, D) R \exp (-\beta R)(u-v) .
\end{aligned}
$$

Given an arbitrary $\epsilon>0$, there exists an $R_{1}$ such that, for all $R>R_{0}, R_{1}$ and $r_{1} \leqq r \leqq r_{2}$, the right-hand member of (13) is less than $\epsilon$. That is

$$
\left|\int_{C} \frac{a(\omega) z^{\omega}}{\exp (2 \pi i \omega)-1} d \omega\right|<\epsilon, \quad R>R_{0}, R_{1} ; r_{1} \leqq r \leqq r_{2} .
$$

By (14) it follows that the integral along the arc converges uniformly to zero as $R$ becomes infinite. Hence by (3) and (4) we may write

$$
\sum_{n=l}^{\infty} a(n) z^{n}=\int_{I_{2}} \frac{a(\omega) z^{\omega}}{\exp (2 \pi i \omega)-1} d \omega+\int_{I_{1}} \frac{a(\omega) z^{\omega}}{\exp (2 \pi i \omega)-1} d \omega .
$$

Let

$$
J_{\psi_{1}}(z)=\int_{I_{1}} \frac{a(\omega) z^{\omega}}{\exp (2 \pi i \omega)-1} d \omega
$$

and

$$
J_{\psi_{2}}(z)=\int_{I_{2}} \frac{a(\omega) z^{\omega}}{\exp (2 \pi i \omega)-1} d \omega .
$$

It will be shown that $J_{\psi_{1}}(z)+J_{\psi_{2}}(z)$ is regular for $z$ in a domain 
which contains the interval $-r_{2} \leqq z \leqq-r_{1}$ in its interior.

In this discussion four cases with regard to the angles $\psi_{1}$ and $\psi_{2}$ present themselves naturally.
A:
$-\pi / 2<\psi_{2}<0$,
$0<\psi_{1}<\pi / 2$
B:
$-\pi / 2<\psi_{2}<0$,
$\psi_{1}=\pi / 2$
C:
$\psi_{2}=-\pi / 2$,
$\psi_{1}=\pi / 2$
D:

$$
-\pi<v \leqq \psi_{2} \leqq-\pi / 2,
$$$$
\pi / 2<\psi_{1} \leqq v<\pi \text {. }
$$

Consider case A. Here

$$
\begin{aligned}
J_{\psi_{1}}(z) & =\int_{I_{1}} \frac{a(\omega) z^{\omega}}{\exp (2 \pi i \omega)-1} d \omega \\
& =-\int_{0}^{\infty} \frac{a(\omega) \exp \left[\left(h+R \exp \left(i \psi_{1}\right)\right)(\log r+i \theta)\right]}{\exp (2 \pi i \omega)-1} \exp \left(i \psi_{1}\right) d R .
\end{aligned}
$$

From (5) and (6) it follows that

$$
\left|J_{\psi_{1}}(z)\right|<A B r^{h} \int_{0}^{\infty} R^{K} \exp \left[-R\left(\log r^{-1} \cos \psi_{1}+\theta \sin \psi_{1}\right)\right] d R .
$$

If

$$
0<r<\exp \left(\theta \tan \psi_{1}\right), \quad 0 \leqq \theta<2 \pi,
$$

then $b\left(r, \theta, \psi_{1}\right)=\log r^{-1} \cos \psi_{1}+\theta \sin \psi_{1}>0$.

Suppose $z=r \exp (i \theta)$ is in some closed domain ${ }^{3}$ contained in the domain defined by (17). Denote by $b^{\prime}\left(\psi_{1}\right)>0$ the minimum assumed by $b\left(r, \theta, \psi_{1}\right)$ in this closed domain. Then (16) becomes

$$
\left|J_{\psi_{1}}(z)\right|<A B r^{h} \int_{0}^{\infty} R^{K} \exp \left[-b^{\prime}\left(\psi_{1}\right) R\right] d R<M,
$$

where $M$ is a constant.

Therefore $J_{\psi_{1}}(z)$ converges uniformly for $z$ in any closed domain contained in (17). For $\omega$ on $I_{1}$ and $z$ contained in the domain defined by (17) the integrand of $J_{\psi_{1}}(z)$ is continuous in $\omega$ and $z$. It follows from our definition of $z^{\omega}$ that for a fixed $\omega$ on $I_{1}$ the integrand is a regular function of $z$ for $z$ in any closed domain contained in (17). Then by well known theorems [2], $J_{\psi_{1}}(z)$ is regular for $z$ in any closed domain contained in (17).

\section{Consider}

${ }^{3} D$ is said to be a closed domain if there exists a domain $E$ with the property that $D=E$. Here if $E$ is a given set and $E^{\prime}$ its derived set then $E=E+E^{\prime}$. 


$$
\begin{aligned}
J_{\psi_{2}}(z) & =\int_{I_{2}} \frac{a(\omega) z^{\omega}}{\exp (2 \pi i \omega)-1} d \omega \\
& =\int_{0}^{\infty} \frac{a(\omega) \exp \left[\left(h+R \exp \left(i \psi_{2}\right)\right)(\log r+i \theta)\right]}{\exp (2 \pi i \omega)-1} \exp \left(i \psi_{2}\right) d R .
\end{aligned}
$$

By (5) and (7) we may write

$$
\begin{aligned}
& \left|J_{\psi_{2}}(z)\right| \\
& \quad<A D r^{h} \int_{0}^{\infty} R^{K} \exp \left[-R \log r^{-1} \cos \psi_{2}+(\theta-2 \pi) \sin \psi_{2}\right] d R .
\end{aligned}
$$

If

$$
0<r<\exp \left[(\theta-2 \pi) \tan \psi_{2}\right]
$$

then $b\left(r, \theta, \psi_{2}\right)=\log r^{-1} \cos \psi_{2}+(\theta-2 \pi) \sin \psi_{2}>0$.

Let $z=r \exp (i \theta)$ be in any closed domain contained in the domain defined by (19). Denote by $b^{\prime}\left(\psi_{2}\right)>0$ the minimum taken on by $b\left(r, \theta, \psi_{2}\right)$ in this closed domain; then (18) becomes

$$
\left|J_{\psi_{2}}(z)\right|<A D r^{h} \int_{0}^{\infty} R^{K} \exp \left[-b^{\prime}\left(\psi_{2}\right) R\right] d R<N,
$$

where $N$ is a constant.

It then follows by the same analysis employed in the case of $J_{\psi_{1}}(z)$ that $J_{\psi_{2}}(z)$ is regular in any closed domain contained in the domain defined by (19). The function $J_{\psi_{1}}(z)+J_{\psi_{2}}(z)$ will therefore be regular for $z=r \exp (i \theta)$ in any closed domain contained in the domain common to (17) and (19).

It has been shown (15) that

$$
\sum_{n=l}^{\infty} a(n) z^{n}=J_{\psi_{1}}(z)+J_{\psi_{2}}(z)
$$

where $z=r \exp (i \pi)$ and $r_{1} \leqq r \leqq r_{2}$. But $J_{\psi_{1}}(z)+J_{\psi_{2}}(z)$ has been shown to be regular in a domain which includes the interval $-r_{2} \leqq z \leqq-r_{1}$ in its interior. Hence $J_{\psi_{1}}(z)+J_{\psi_{2}}(z)$ provides the analytic continuation of the function defined by $\sum_{n=l}^{\infty} a(n) z^{n}$ to any closed domain contained in the domain common to (17) and (19). Since

$$
f(z)=\sum_{n=0}^{l-1} a(n) z^{n}+\sum_{n=l}^{\infty} a(n) z^{n},
$$

it is evident that $J_{\psi_{1}}(z)+J_{\psi_{2}}(z)+\sum_{n=0}^{l-1} a(n) z^{n}$ provides the analytic continuation of $f(z)$ to the same domain. Hence in this case $f(z)$ will 
in general be regular in a domain bounded by two spirals as indicated in Figure 2.

Consider case $\mathrm{B}$. By an analysis similar to that given in case $\mathrm{A}$ it is easily shown that $J_{\psi_{1}}(z)+J_{\psi_{2}}(z)$ is regular in any closed domain contained in the domain common to

$$
0<r<\exp \left[(\theta-2 \pi) \tan \psi_{2}\right], \quad 0 \leqq \theta<2 \pi,
$$

and the whole complex plane excluding the segment 1 to $+\infty$. By an

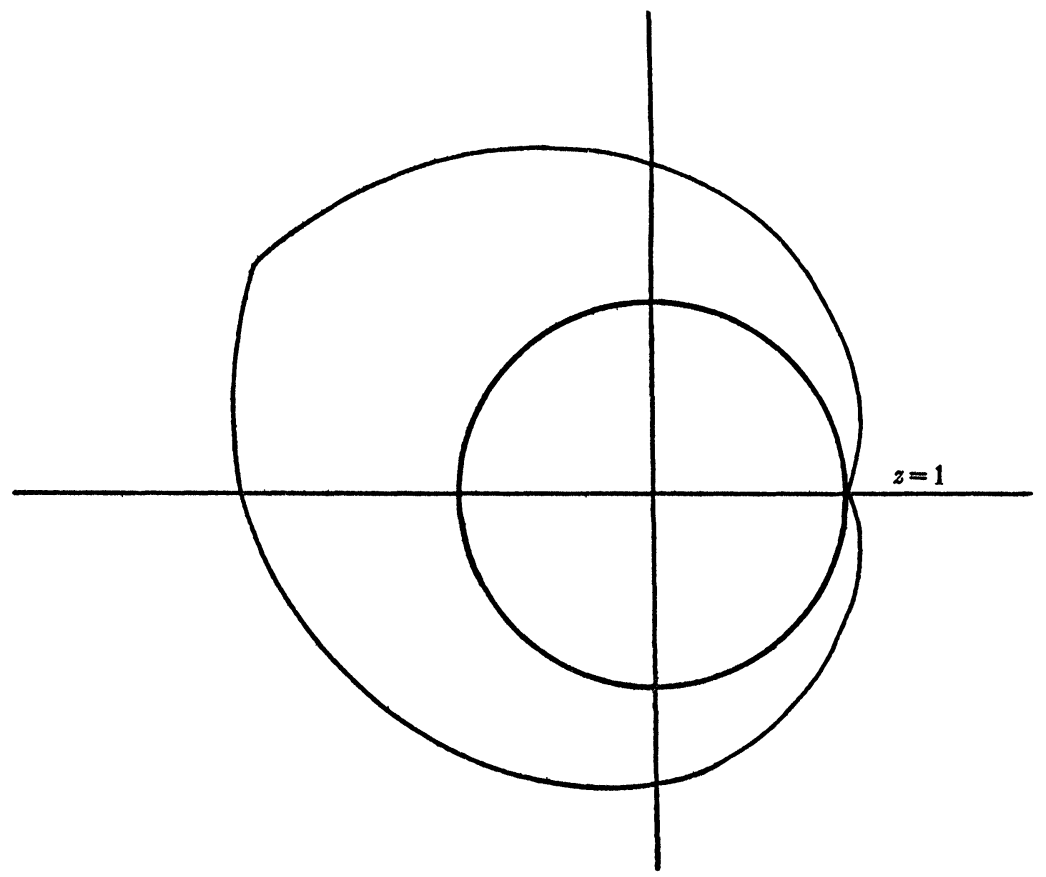

F1G. 2

argument similar to that employed in case $\mathrm{A}$ it is easily seen that $f(z)$ is regular in any closed domain contained in the domain common to (20) and the whole complex plane excluding the segment 1 to $+\infty$.

Case C. Here we have a very special case of the theorem of LeRoy and Lindelöf [1]. It could be shown by an analysis similar to the preceding that $f(z)$ is regular in any domain of the complex plane excluding the segment 1 to $+\infty$.

Finally we consider case D. By a method similar to that employed in case $\mathrm{A}$ it is simple to show that $J_{\psi_{1}}(z)$ converges uniformly for $z$ in any closed bounded domain contained in the domain defined by 


$$
r>\exp \left(\theta \tan \psi_{1}\right),
$$$$
0 \leqq \theta<2 \pi,
$$

and that $J_{\psi_{2}}(z)$ converges uniformly for $z$ in any closed bounded domain contained in the domain defined by

$$
r>\exp \left[(\theta-2 \pi) \tan \psi_{2}\right], \quad 0 \leqq \theta<2 \pi \text {. }
$$

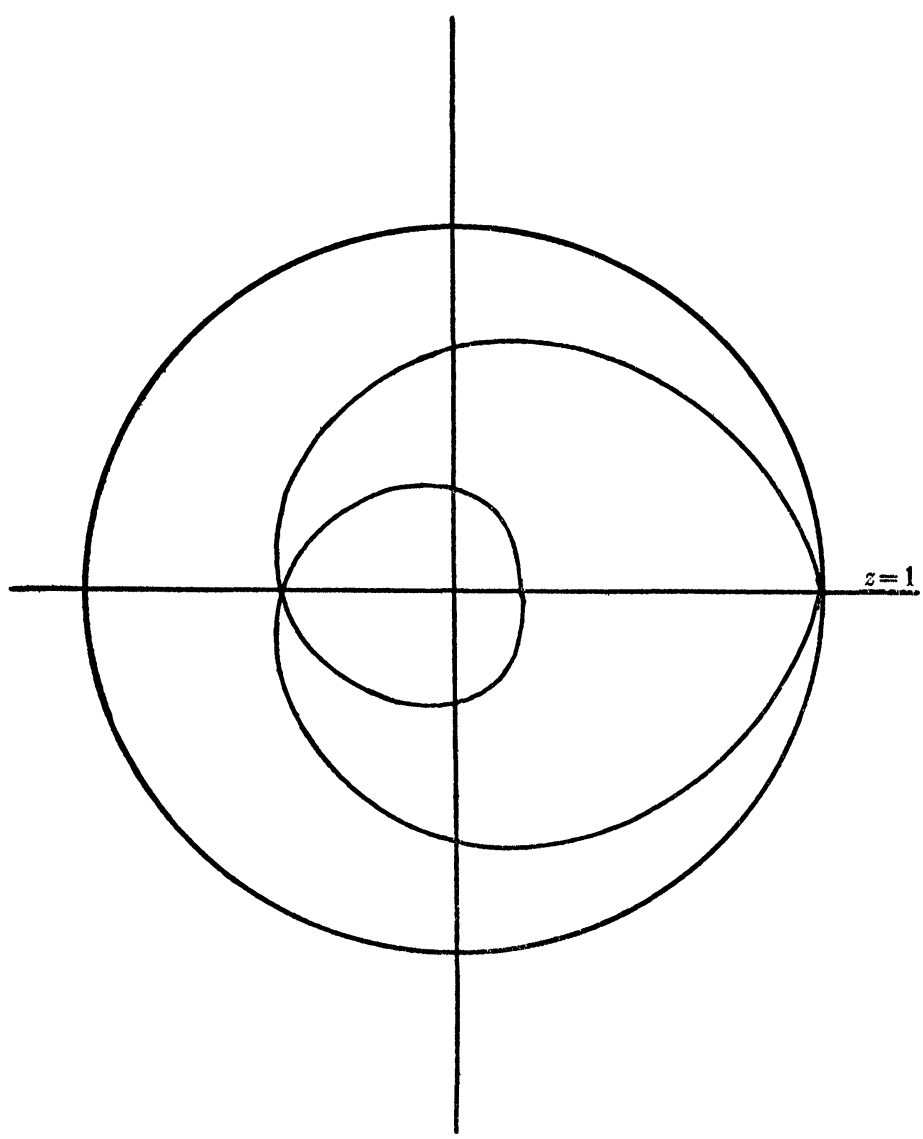

FIG. 3

Now the domain common to (21) and (22) contains the segment $-r_{2} \leqq z \leqq-r_{1}$ in its interior. This is easily seen by setting $\theta=\pi$ in (21) and (22) and noting that $r_{1}$ is the larger of $\exp (\pi \tan u)+\alpha$ and $\exp (\pi \tan v)+\alpha, \alpha>0$. Hence by arguments similar to those used in case A

$$
J_{\psi_{1}}(z)+J_{\psi_{2}}(z)+\sum_{n=0}^{l-1} a(n) z^{n}
$$


provides the analytic continuation of $f(z)$ to any closed bounded domain contained in the domain common to (21) and (22). For the case $\psi_{1}=\psi_{2}$ the spirals (21) and (22) are indicated in Figure 3. In calculating $J_{\psi_{1}}(z)$ and $J_{\psi_{2}}(z)$ for a given $z=r \exp (i \theta)$ we recall that by our convention

$$
\boldsymbol{z}^{\omega}=\exp [\omega(\log r+i \theta)], \quad 0 \leqq \theta<2 \pi .
$$

Hence we have the following theorem.

THEOREM 1. Let $f(z)=\sum_{n=0}^{\infty} a_{n} z^{n}$ with radius of convergence unity. Let the coefficients $a_{n}$ be the values taken on by an analytic function $a(z)$ at $z=0,1,2, \cdots$. Suppose $a(z)$ is regular with the possible exception of $a$ pole of order $K$ at infinity, in an angle with vertex $h>0$ (non-integral) on the axis of reals and including the axis of positive reals in its interior. Let the sides of this angle make angles $\psi_{1}$ and $\psi_{2}$ with the axis of reals. Then, if $\gamma>0$ but otherwise arbitrarily small, and

$$
0<\psi_{1}<\pi / 2, \quad-\pi / 2<\psi_{2}<0,
$$

$f(z)$ is regular in the domain common to

$$
r \leqq \exp \left[\theta \tan \psi_{1}\right]-\gamma, \quad 0 \leqq \theta<2 \pi,
$$

and

$$
r \leqq \exp \left[(\theta-2 \pi) \tan \psi_{2}\right]-\gamma, \quad 0 \leqq \theta<2 \pi .
$$

If

B: $\quad \psi_{1}=\pi / 2, \quad-\pi / 2<\psi_{2}<0$,

$f(z)$ is regular in any closed domain common to

$$
r \leqq \exp \left[(\theta-2 \pi) \tan \psi_{2}\right]-\gamma, \quad 0 \leqq \theta<2 \pi,
$$

and the whole complex plane excluding the segment 1 to $+\infty$. If

C:

$$
\psi_{1}=\pi / 2, \quad \psi_{2}=-\pi / 2,
$$

$f(z)$ is regular in any closed domain of the complex plane excluding the segment 1 to $+\infty$. If

$\mathrm{D}: \quad \pi / 2<\psi_{1} \leqq u<\pi, \quad-\pi<v \leqq \psi_{2}<-\pi / 2$,

$f(z)$ is regular in any bounded domain common to

$$
r \geqq \exp \left(\theta \tan \psi_{1}\right)+\gamma, \quad 0 \leqq \theta<2 \pi,
$$

and 


$$
r \geqq \exp \left[(\theta-2 \pi) \tan \psi_{2}\right]+\gamma, \quad 0 \leqq \theta<2 \pi .
$$

It is clear ${ }^{4}$ that the theorem above is still true even though $a(z)$ does not have a pole at infinity. All that is necessary is that $a(z)$ be single-valued in the angle and that $|a(h+R \exp (i \psi))|\left\langle A R^{K}, R>R_{0}\right.$.

3. $a(z)$ may have an essential singularity at infinity. Suppose $a(z)$ is regular interior to and on the sides of the angular opening of Figure 1. Suppose there exists an $R_{0}$ such that for $R>R_{0}$ and $z=h$ $+R \exp (i \psi)$ in this angle

$$
|a(h+R \exp (i \psi))|<\exp (\delta R) .
$$

In order to simplify the work to follow suppose $\delta \leqq \pi-d, d>0$.

It will be shown that, if we place certain restrictions on $\psi_{1}, \psi_{2}$ and $z$,

$$
\int_{C} \frac{a(\omega) z^{\omega}}{\exp (2 \pi i \omega)-1} d \omega
$$

converges uniformly to zero as $R$ becomes infinite. Let $z=r \exp (i \pi)$ and $\omega=h+R \exp (i \psi)$ with $-\pi / 2 \leqq \psi \leqq \pi / 2$.

If $r<\exp [\pi \tan \psi-\delta \sec \psi]$, then

$$
b_{1}(r, \psi)=\log r^{-1} \cos \psi+\pi \sin \psi-\delta>0 .
$$

If $r<\exp [-\pi \tan \psi-\delta \sec \psi]$, then

$$
b_{2}(r, \psi)=\log r^{-1} \cos \psi-\pi \sin \psi-\delta>0 .
$$

Set $g(\psi)=\pi \tan \psi-\delta \sec \psi$ where $0 \leqq \psi \leqq \pi / 2$ and $\delta \leqq \pi-d, d>0$. Now $g^{\prime}(\psi)=\sec \psi(\pi \sec \psi-\delta \tan \psi)$. Hence for $\delta \leqq \pi-d$ and $0 \leqq \psi$ $<\pi / 2, g^{\prime}(\psi)$ is positive.

As $\psi$ approaches $\pi / 2, g(\psi)$ approaches $+\infty$. Hence the minimum value of $g(\psi)$ on the interval $0 \leqq \psi \leqq \pi / 2$ is $g(0)$, that is $-\delta$. In a similar manner we see that the minimum of $-\pi \tan \psi-\delta \sec \psi$ on the interval $-\pi / 2 \leqq \psi \leqq 0$ is $-\delta$.

Hence if $r<\exp [-\delta-p]$ where $p>0$ but otherwise arbitrarily small, (25) holds for $0 \leqq \psi \leqq \pi / 2$ and (26) holds for $-\pi / 2 \leqq \psi \leqq 0$.

Denote by $b_{1}^{\prime}>0$ the minimum assumed by $b_{1}(r, \psi)$ on the interval $0 \leqq \psi \leqq \pi / 2$ and by $b_{2}^{\prime}>0$ the minimum assumed by $b_{2}(r, \psi)$ on the interval $-\pi / 2 \leqq \psi \leqq 0$ where $0 \leqq r \leqq \exp [-\delta-p], p>0$. Let $b_{0}>0$ be the smaller of $b_{1}^{\prime}>0$ and $b_{2}^{\prime}>0$.

Then from (6), (7) and (23) we have

\footnotetext{
4 The author is indebted to the referee for this observation.
} 


$$
\begin{aligned}
\left|\int_{C} \frac{a(\omega) z^{\omega} d \omega}{\exp (2 \pi i \omega)-1}\right| & \leqq R r^{h} T_{1}(B, D) \int_{-\pi / 2}^{\pi / 2} \exp \left[-b_{0} R\right] d \psi \\
& =\operatorname{Rr}^{h} T_{1}(B, D) \exp \left[-b_{0} R\right] \pi
\end{aligned}
$$

Given an $\epsilon>0$ we can choose an $R_{1}$ such that for $R>R_{0}, R_{1}$ and $0 \leqq r \leqq \exp [-\delta-p], p>0$, the quantity on the right of (27) is less than $\epsilon$. That is, (24) converges uniformly to zero as $R$ becomes infinite.

Let $\pi / 2 \leqq \psi \leqq u<\pi$. If $r>\exp [\pi \tan \psi-\delta \sec \psi]$ then

$$
b_{1}(r, \psi)=\log r^{-1} \cos \psi+\pi \sin \psi-\delta>0 .
$$

Let $-\pi<v \leqq \psi \leqq-\pi / 2$. If $r>\exp [-\pi \tan \psi-\delta \sec \psi]$ then

$$
b_{2}(r, \psi)=\log r^{-1} \cos \psi+\pi \sin \psi-\delta>0 .
$$

Denote, for a given $q>0$ but otherwise arbitrarily small, by $F$ the smaller of the numbers $\pi \sin u-q$ and $\pi \sin v-q$. Let $\delta \leqq F$. The maximum for $\pi / 2 \leqq \psi \leqq u<\pi$ of $\pi \tan \psi-\delta \sec \psi$ is $\pi \tan \psi-F \sec \psi$. The maximum for $-\pi<v \leqq \psi \leqq-\pi / 2$ of $-\pi \tan \psi-\delta \sec \psi$ is $\pi \tan v$ $-F \sec v$. Denote by $E$ the larger of $\pi \tan u-F \sec u+t$ and $\pi \tan v-F \sec v+t$ where $t>0$ but sufficiently small that $E<0$. If $r>\exp (E)$ then (28) and (29) hold. Suppose now in addition that $u$ and $v$ have the property that

$$
\cos u>-\frac{\pi \sin u-F}{\delta+p+t}
$$

and

$$
\cos v>-\frac{\pi \sin v-F}{\delta+p+t} .
$$

Here $p$ and $t$ are positive but otherwise may be chosen arbitrarily small. Let $z=r \exp (i \pi)$ with

$$
\exp E \leqq r \leqq \exp (-\delta-p) .
$$

That there is an interval consisting of more than one point satisfying (32) follows from the restrictions (30) and (31) placed on $u$ and $v$. For if $u$ and $v$ satisfy (30) and (31) then $E<-\delta-p$. Denote by $b_{1}^{\prime \prime}>0$ the minimum assumed by $b_{1}(r, \psi)$ on $\pi / 2 \leqq \psi \leqq u<\pi$ and by $b_{2}^{\prime \prime}>0$ the minimum assumed by $b_{2}(r, \psi)$ on $-\pi<v \leqq \psi \leqq-\pi / 2$ where $r$ satisfies (32). Denote by $b_{0}^{\prime \prime}>0$ the smaller of $b_{1}^{\prime \prime}$ and $b_{2}^{\prime \prime}$. Let $b^{*}>0$ be the smaller of $b_{0}$ and $b_{0}^{\prime \prime}$. Then by (6), (7) and (23) if $-\pi<v \leqq \psi \leqq u<\pi, \delta \leqq F$ and $\exp E \leqq r \leqq \exp (-\delta-p)$, where $u$ and $v$ satisfy (30) and (31) we have 


$$
\begin{aligned}
\left|\int_{C} \frac{a(\omega) z^{\omega}}{\exp (2 \pi i \omega)-1} d \omega\right| & \leqq R^{h} T_{2}(B, D) \int_{v}^{u} \exp \left(-b^{*} R\right) d \psi \\
& \leqq R^{h} T_{2}(B, D) \exp \left(-b^{*} R\right)(u-v) .
\end{aligned}
$$

Given an $\epsilon>0$ there exists an $R_{1}$ such that for $R>R_{0}, R_{1}$ and $\exp E \leqq r \leqq \exp (-\delta-p)$ the quantity on the right of (33) is less than $\epsilon$. Hence as $R$ becomes infinite we again obtain (15) since (24) converges uniformly to zero.

It is now possible to consider again the four cases of $\$ 2$; however, for brevity we shall consider only those corresponding to A and D. Let us denote these by $A^{\prime}$ and $D^{\prime}$.

$\mathrm{A}^{\prime}$ :

$$
\begin{aligned}
& 0<\psi_{1}<\pi / 2, \quad-\pi / 2<\psi_{2}<0, \\
& \pi / 2<\psi_{1} \leqq u \text {, } \\
& v \leqq \psi_{2}<-\pi / 2 \text {. }
\end{aligned}
$$

$\mathrm{D}^{\prime}$ :

Case $\mathrm{A}^{\prime}$. Suppose that $\delta \leqq \pi-d, d>0$. It will be shown that $J_{\psi_{1}}(z)+J_{\psi_{2}}(z)$ is regular for $z$ in a domain which includes all or part of the segment $-\exp [-\delta-p] \leqq z<0$ in its interior. By a method similar to that employed in $\$ 2$ we could show that for $J_{\psi_{1}}(z)$ to converge for a fixed $z=r \exp (i \theta)$ it is sufficient that

$$
0<r<\exp \left[\theta \tan \psi_{1}-\delta \sec \psi_{1}\right], \quad 0 \leqq \theta<2 \pi .
$$

It follows then that $J_{\psi_{1}}(z)$ will converge uniformly for $z$ in any closed domain contained in the domain defined by (34). Hence by reasoning similar to that employed in $\$ 2$, it is easily seen that $J_{\psi_{1}}(z)$ is regular for $z$ in any closed domain contained in (34).

Consider $J_{\psi_{2}}(z)$. It is easily shown that it converges for a fixed $z=r \exp (i \theta)$ contained in the domain defined by

$$
0<r<\exp \left[(\theta-2 \pi) \tan \psi_{2}-\delta \sec \psi_{2}\right], \quad 0 \leqq \theta<2 \pi .
$$

If then $z=r \exp (i \theta)$ is in any closed domain contained in the domain defined by (35), $J_{\psi_{2}}(z)$ converges uniformly and hence represents a regular function. The function $J_{\psi_{1}}(z)+J_{\psi_{2}}(z)$ will therefore be regular in any closed domain contained in the domain common to (34) and (35). We have seen that if $z=r \exp (i \pi)$ with $\exp E \leqq r \leqq \exp [-\delta-p]$, $p>0$, that

$$
\sum_{n=l}^{\infty} a(n) z^{n}=J_{\psi_{1}}(z)+J_{\psi_{2}}(z)
$$

But $J_{\psi_{1}}(z)+J_{\psi_{2}}(z)$ has been shown to be regular in a domain which includes all or part of the interval exp $E \leqq r \leqq \exp [-\delta-p]$ in its interior. Therefore $J_{\psi_{1}}(z)+J_{\psi_{2}}(z)+\sum_{n=0}^{l-1} a(n) z^{n}$ provides the analytic 
continuation of $f(z)$ to any closed domain contained in the domain common to (34) and (35). Hence in this case $f(z)$ will be regular in a domain bounded by two spirals as indicated in Figure 4.

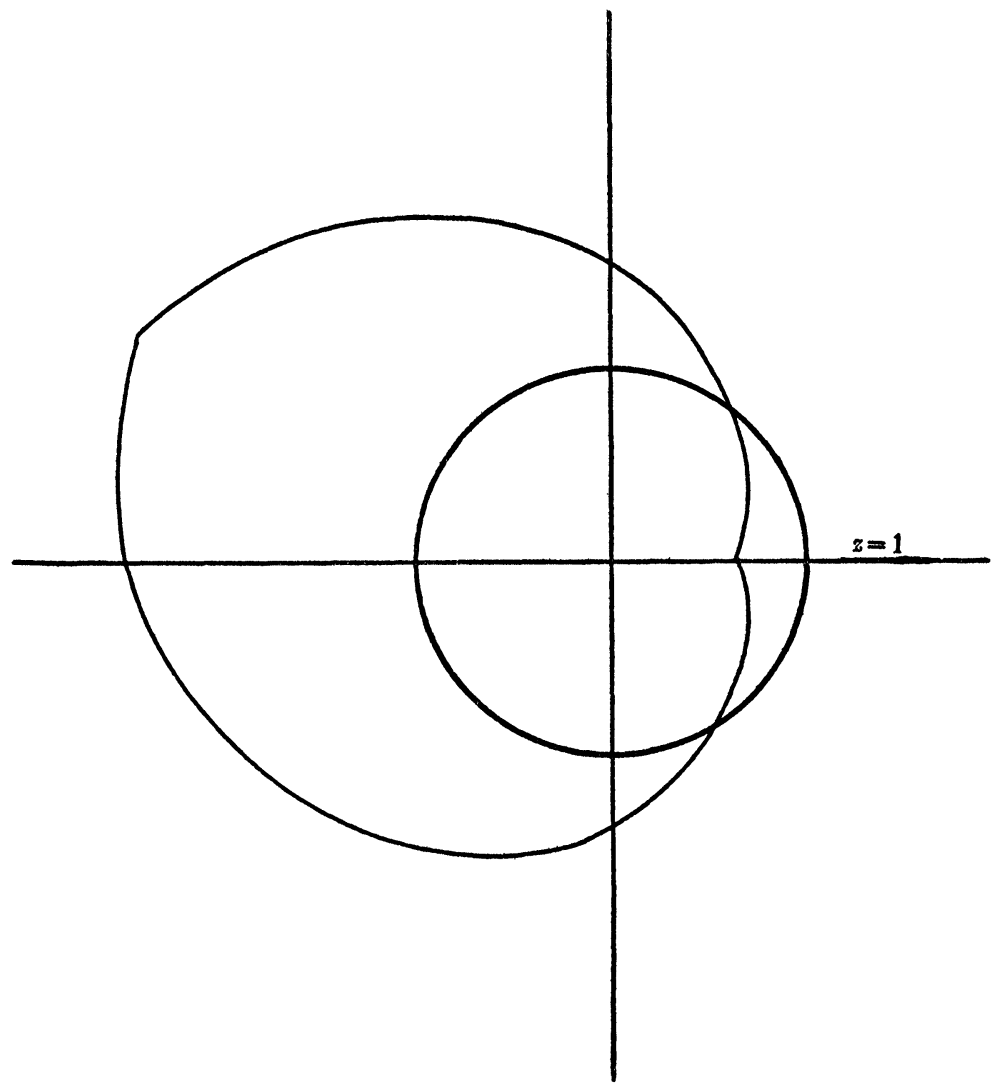

FIG. 4

Consider case $\mathrm{D}^{\prime}$. Suppose $\delta \leqq F$ and that $v$ and $u$ satisfy (30) and (31). Then for exp $E \leqq r \leqq \exp (-\delta-p)$ it follows from (33) that (15) holds. It is easily seen that $J_{\psi_{1}}(z)$ will converge for a fixed $z=r \exp (i \theta)$ if $\log r^{-1} \cos \psi_{1}+\theta \sin \psi_{1}-\delta>0$. This will be the case if

$$
r>\exp \left[\theta \tan \psi_{1}-\delta \sec \psi_{1}\right], \quad 0 \leqq \theta<2 \pi .
$$

It is evident that $J_{\psi_{1}}(z)$ will converge uniformly for $z=r \exp (i \theta)$ in any closed bounded domain contained in the domain defined by (36). In order that $J_{\psi_{2}}(z)$ converge for a fixed $z=r \exp (i \theta)$ it is sufficient that $\log r^{-1} \cos \psi_{2}+(\theta-2 \pi) \sin \psi_{2}-\delta>0$. That is,

$$
r>\exp \left[(\theta-2 \pi) \tan \psi_{2}-\delta \sec \psi_{2}\right], \quad 0 \leqq \theta<2 \pi .
$$


Then $J_{\psi_{2}}(z)$ will converge uniformly for $z=r e^{i \theta}$ in any closed bounded domain contained in the domain defined by (37). Hence

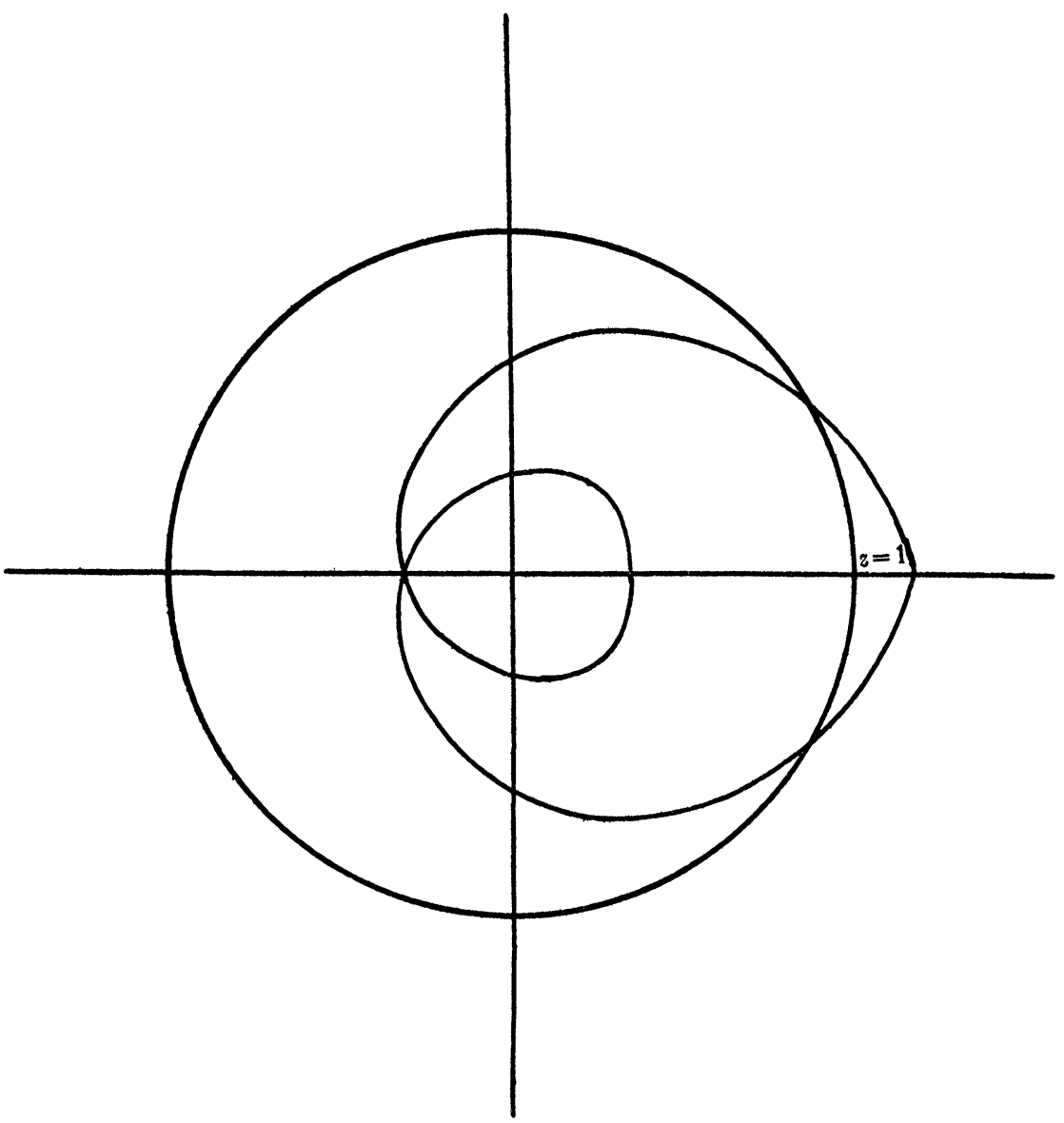

Fig. 5

$J_{\psi_{1}}(z)+J_{\psi_{2}}(z)$ will converge uniformly for $z=r e^{i \theta}$ in any closed bounded domain contained in the domain common to (36) and (37). It is evident that the region common to (36) and (37) contains the interval $-\exp (-\delta-p) \leqq z \leqq-\exp E$ in its interior. For $E$ is by definition the larger of $\pi \tan u-F \sec u+t$ and $\pi \tan v-F \sec v+t, t>0$, and if we set $\theta=\pi$ in (36) and (37) it is clear that both exponents are smaller than $E$. Therefore $J_{\psi_{1}}(z)+J_{\psi_{2}}(z)+\sum_{n=0}^{b-1} a(n) z^{n}$ provides the analy tic continuation of $f(z)$ to any closed bounded domain contained in the domain common to (36) and (37). For the case $\psi_{1}=\psi_{2}$ this will be such a domain as indicated in Figure 5 . 
Hence we have the following theorem.

THEOREM 2. Let $f(z)=\sum_{n=0}^{\infty} a_{n} z^{n}$ with radius of convergence unity. Let the coefficients $a_{n}$ be the values taken on by an analytic function $a(z)$ at $z=0,1,2, \cdots$. Suppose $a(z)$ is regular with the possible exception of an essential singularity at infinity in an angle with vertex $h>0$ (nonintegral) on the real axis, including the axis of positive reals in its interior. Let the sides of this angle make angles $\psi_{1}$ and $\psi_{2}$ with the axis of positive reals. Then if, for $z=h+R \exp (i \psi)$ in this angular opening, $a(z)$ satisfies the inequality

$$
|a(h+R \exp (i \psi))|<\exp (\delta R), \quad \quad R>R_{0},
$$

where $\delta \leqq \pi-d, d>0$, and

$\mathrm{A}^{\prime}$ :

$$
0<\psi_{1}<\pi / 2, \quad-\pi / 2<\psi_{2}<0,
$$

$f(z)$ is regular in the domain common to

$$
r \leqq \exp \left[\theta \tan \psi_{1}-\delta \sec \psi_{1}\right]-\gamma, \quad \gamma>0
$$

and

$$
r \leqq \exp \left[(\theta-2 \pi) \tan \psi_{2}-\delta \sec \psi_{2}\right]-\gamma, \quad \gamma>0,
$$

for $0 \leqq \theta<2 \pi$.

For a given $q>0$ but otherwise arbitrarily small let $F$ be the smaller of the numbers $\pi \sin u-q$ and $\pi \sin v-q$. Suppose $u$ and $v$ may be chosen such that for a given $p$ and $t$ positive but otherwise arbitrarily small

$$
\cos u>-\frac{\pi \sin u-F}{\delta+p+t}
$$

and

$$
\cos v>-\frac{\pi \sin v-F}{\delta+p+t}
$$

Then if $\delta \leqq F$ and

$\mathrm{D}^{\prime}: \quad \pi / 2<\psi_{1} \leqq u<\pi, \quad-\pi<v \leqq \psi_{2}<-\pi / 2$,

$f(z)$ is regular in any bounded domain common to

$$
r \geqq \exp \left[\theta \tan \psi_{1}-\delta \sec \psi_{1}\right]+\gamma, \quad \gamma>0,
$$

and

$$
r \geqq \exp \left[(\theta-2 \pi) \tan \psi_{2}-\delta \sec \psi_{2}\right]+\gamma, \quad \gamma>0,
$$

where $0 \leqq \theta<2 \pi$. 
4. Conclusions. We note first that if, in Theorem $1, \psi_{1}>0$ and $\psi_{2}<0$ but otherwise arbitrarily small, that $f(z)$ has $z=1$ as its only singularity on the circle of convergence.

In part $\mathrm{D}$ of Theorem 1 if both $\psi_{1}$ and $\psi_{2}$ are greater than $90^{\circ}$ in magnitude, that is, the sector of regularity is greater than $180^{\circ}$, we have the rather remarkable result that $z=1$ is the only singularity of $f(z)$ in the finite plane. Thus for example the function defined by the series

$$
\sum_{n=0}^{\infty} \frac{z^{n}}{(n+\alpha)^{\beta}}
$$

where $\beta$ is an integer and $\alpha$ is not equal to zero or a negative integer but otherwise arbitrary, has the point $z=1$ as its only singularity in the finite plane.

It is of course clear that we may use the results obtained in a different manner, that is, if $f(z)$ has a singular point on the circle of convergence other than $z=1$ then $a(z)$ cannot be analytic in an angular opening including the axis of positive reals in its interior with at most a pole of finite order at infinity.

If the inequality for $a(z)$ in Theorem 2 is satisfied for every $\delta>0$ however small, then under the condition of case $\mathrm{D}^{\prime}$ of Theorem 2, $z=1$ is the only singularity in the finite plane. This result $t^{5}$ is analogous to the following theorem due to Faber [1].

THEOREM (FABER). If $g(z)$ is an integral function such that $\left|g\left(r e^{i \theta}\right)\right|<e^{e r}$ for an arbitrary positive $\epsilon$ and $r>r^{\prime}$, the function $f(z) d e-$ fined by $\sum_{n=0}^{\infty} g(n) z^{n}$ and its analytic continuation has the point 1 as its only singular point.

We observe now that the bounding curves

$$
r<\exp \left[\theta \tan \psi_{1}-\delta \sec \psi_{1}\right], \quad 0 \leqq \theta<2 \pi,
$$

and

$$
r<\exp \left[(\theta-2 \pi) \tan \psi_{2}-\delta \sec \psi_{2}\right], 0 \leqq \theta<2 \pi,
$$

of (34) and (35) cut the unit circle at the points $\exp \left[i \delta \csc \psi_{1}\right]$ and $\exp \left[i\left(2 \pi+\delta \csc \psi_{2}\right)\right]$. If now, in addition to the requirements of part A of Theorem 2, $\psi_{1}, \psi_{2}$ and $\delta$ satisfy the inequality

$$
2 \pi>\delta\left(\csc \psi_{1}-\csc \psi_{2}\right)
$$

it is easily seen that the region common to (34) and (35) will extend beyond the unit circle. We then have the following theorem.

s The author is indebted to the referee for pointing out the analogy. 
THEOREM 3. If the conditions of Theorem 2 part A are satisfied and if in addition the quantities $\psi_{1}, \psi_{2}$ and $\delta$ satisfy the inequality

$$
2 \pi>\delta\left(\csc \psi_{1}-\csc \psi_{2}\right)
$$

then the circle of convergence is not a cut for the function.

\section{BIBLIOGRAPHY}

1. P. Dienes, The Taylor series, Oxford, 1931.

2. E. C. Titchmarsh, The theory of functions, Oxford University Press, 1939, pp. 99-100.

LEHIGH UNIVERSITY

\section{A NOTE ON THE HILBERT TRANSFORM}

\section{LYNN H. LOOMIS}

The Hilbert transform of $f(t),-\infty<t<\infty$, is $1 / \pi$ times the Cauchy principal value

$$
\bar{f}(x)=P \int_{-\infty}^{\infty} \frac{f(t)}{t-x} d t=\lim _{\delta \rightarrow 0+} \int_{\delta}^{\infty} \frac{f(x+t)-f(x-t)}{t} d t .
$$

If $f(t) \in L_{p}, p>1$, then $\bar{f}(x) \in L_{p}$, and a considerable literature is devoted to studying the relationship of such pairs of "conjugate" functions to the theory of functions analytic in a half-plane. More to the point of the present note is a series of papers studying the Hilbert transform along strictly real variable lines $([2,3]$; further bibliography in [2]). ${ }^{1}$

Much less is known about $\bar{f}(x)$ when $f(t) \in L_{1}$. Plessner found by applying complex variable methods to the theory of Fourier series that if $f(t) \in L_{1}$ then $\bar{f}(x)$ exists almost everywhere (see $[1$, p. 145]). Besicovitch [4] proved Plessner's result using only the theory of sets, starting from his own previous real variable investigation of the $L_{2}$ transform case. S. Pollard [5] showed how Besicovitch's proof could be extended to prove the existence a.e. of the principal value of the Stieltjes integral

$$
\bar{f}(x)=P \int_{-\infty}^{\infty} \frac{d F(t)}{t-x},
$$

Received by the editors April 11, 1946.

1 Numbers in brackets refer to the bibliography at the end of the paper. 